\title{
A Review of the Auditory and Non-auditory Effects of Exposure to Noise on Women's Health
}

\author{
Shiva Soury ${ }^{1}$, Rostam Golmohammadi ${ }^{2}$,* \\ ${ }^{1}$ PhD Student, Department of Occupational Health, School of Health, Hamadan University of Medical Sciences, Hamadan, Iran \\ ${ }^{2}$ Professor, Department of Occupational Health, School of Health, Hamadan University of Medical Sciences, Hamadan, Iran \\ * Corresponding Author: Rostam Golmohammadi, Center of Excellence for Occupational Health and Research Center for \\ Health Sciences, School of Health, Hamadan University of Medical Sciences, Hamadan, Iran. Email: golmohamadi@umsha.ac.ir
}

Received: 02/06/2017

Accepted: 24/08/2017

How to Cite this Article: Soury S, Golmohammadi R. A Review of the Auditory and Non-auditory Effects of Exposure to Noise on Women's Health. J Occup Hyg Eng. 2017; 4(2): 53-58. DOI: 10.18 869/acadpub.johe.4.2.53

\begin{abstract}
Background and Objective: Globally, increasing participation of women in the labor market is one of the most important social phenomena. Currently, an exponential number of pregnant mothers are working in industries in the developing countries. In this study, we aimed to analyze all the articles that examined the complications of occupational noise exposure among women. Materials and Methods: The required data were collected from the international databases such as ScienceDirect, Scopus, ISI, and PubMed, and from the national databases including IranMedex, Magiran, and SID during 1980-2016. We performed the search using the following keywords: "occupational exposure", "women", "noise effects", and "pregnancy".

Results: Studies have shown differences in hearing loss and the description of tinnitus between men and women. Some studies have proposed that the effect of noise, as a risk factor for the incidence of ischemic heart disease and changes in plasma cortisol level, is significantly higher in women than in men. In addition, by causing some changes in maternal endocrine system, noise exposure during pregnancy can lead to fetal growth restriction and acquired hearing loss in the baby. Hypertension, painful menstruation, hormonal disorders, and reduced fertility are among other complications of maternal noise exposure.

Conclusion: Exposure to occupational noise, in addition to hearing loss and physiological effects, can have more consequences in women than in men in all circumstances, especially during pregnancy. Therefore, paying attention to the physiological and even psychological characteristics of women, especially pregnant women, in occupational health monitoring programs and periodic medical examinations is of great significance.
\end{abstract}

Keywords: Noise Effects; Occupational Exposure; Pregnancy; Women 


\title{
مرورى بر اثرات شنوايى و غير شنوايى مواجهه شغلى باصدا بر سلامت زنان
}

\author{
شيوا سورى'، رستم كَلمحمدى ז.*

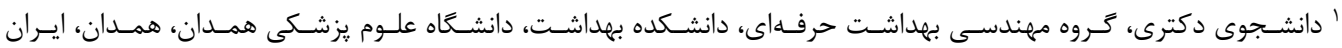

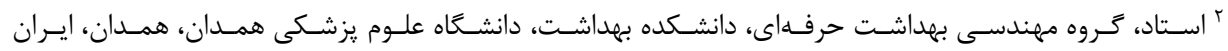

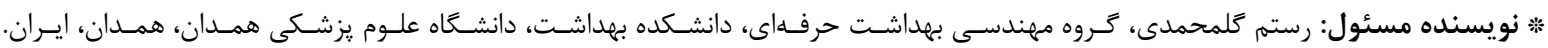 \\ ايميل: golmohamadi@umsha.ac.ir
}

جكيده

سابقه و هدف: امروزه يكى از مهمترين يديدههاى اجتماعى در جهان، افزايش مشاركت زنان در بازار كار

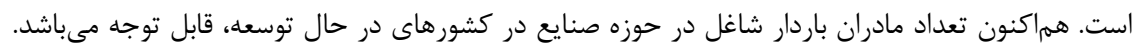

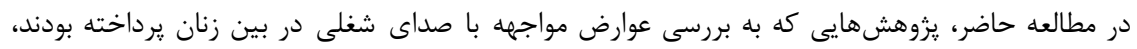

$$
\text { تجزيه و تحليل شد. }
$$

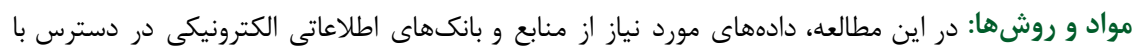

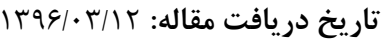

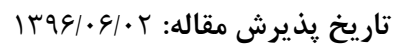

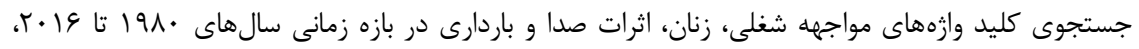
در پايحاههاى اطلاعاتى بينالمللى مانند: Science Direct، Scopus ، تمامى حقوق نشر براى دانشگاه علوم يزشكى همدان محفوظ است.

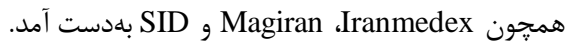
يافتهها: مطالعات انجامشده نشان داد كه تفاوتهايى بين زنان و مردان در بروز افت شنوايى و شرح وزوز

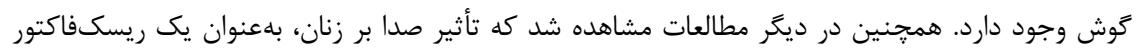

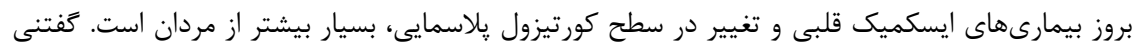

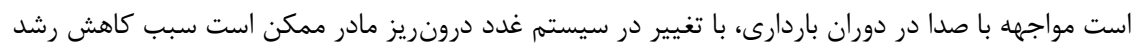

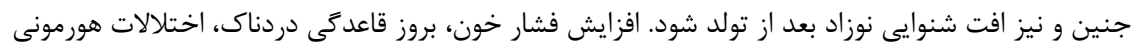

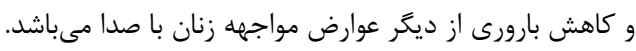

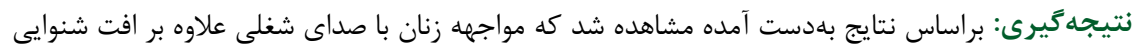

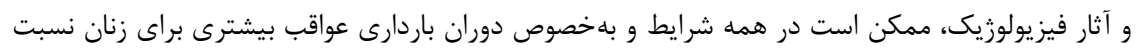

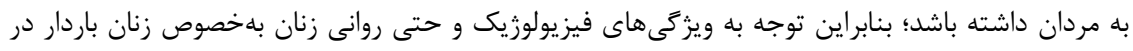

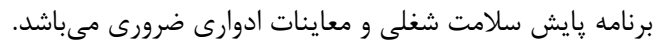

وازَّان كليدى: اثرات صدا، باردارى، زنان، مواجهه شغلى

حدود سه جهارم آنها در آن زمان در سن مؤثر بارورى بودند. در

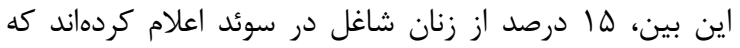

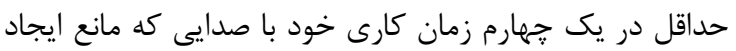

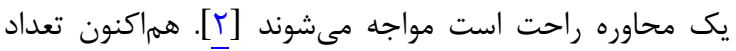

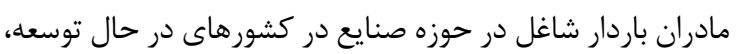
قابل توجه مىباشد؛ بنابراين همواره مسائل مربوط به به عوامل

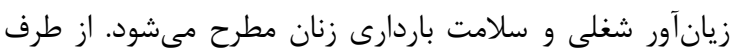

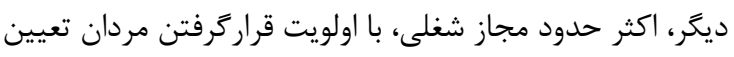

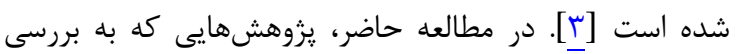
عوارض مواجهه باصداى شغلى در بين زنان يرداخته بودند، تجزيه
امروزه افزايش مشاركت زنان در بازار كار يكى از مهرمترين

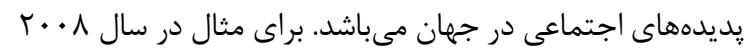

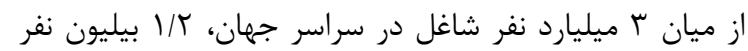

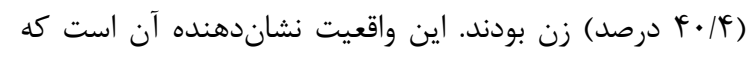

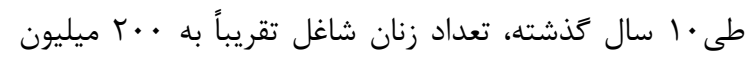

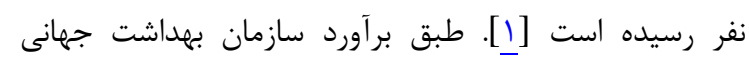

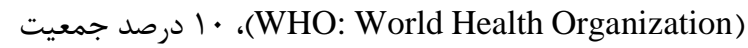

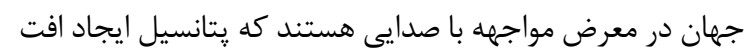

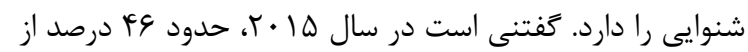

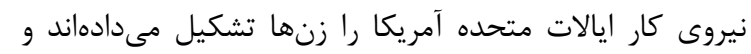


تنش در ناحيه گَردن مىشود. درخور ذكر است در آزمايشهاى

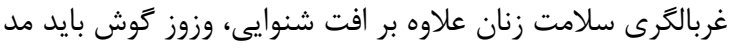

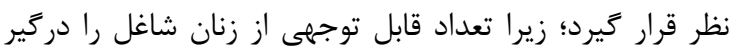

خواهد كرد [111]

\section{استرس فيزيولوزيكس}

نتايج يك مطالعه نشان داد كه خطر آسيبديدكى بوريل براى زنانى

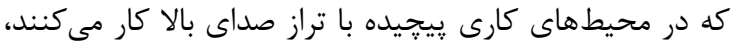

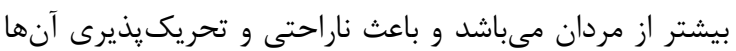

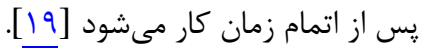

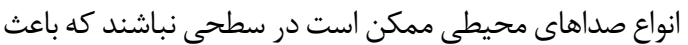

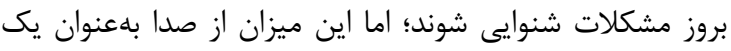

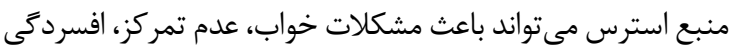

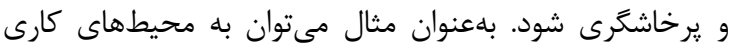

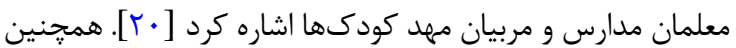

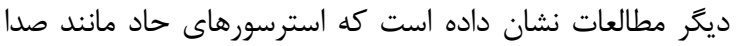

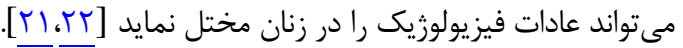

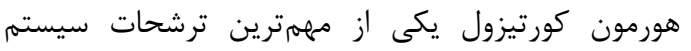

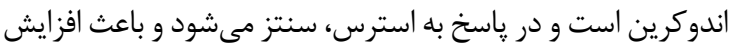

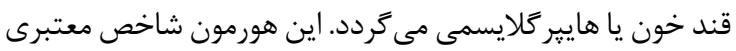

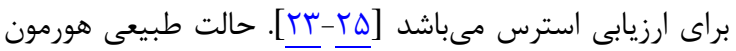

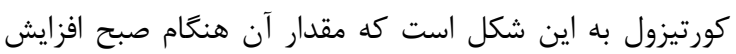

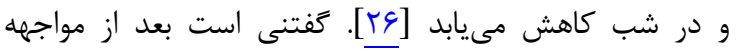

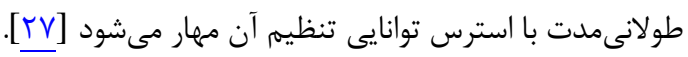

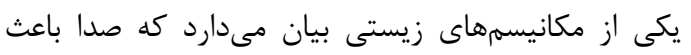

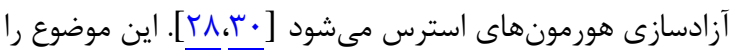

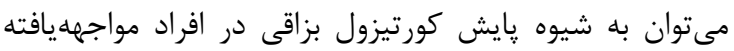

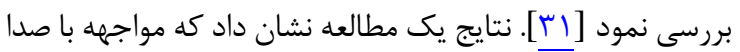
در زنانى كه در نزديكى فرود

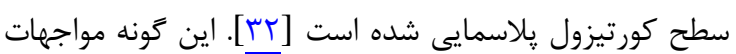

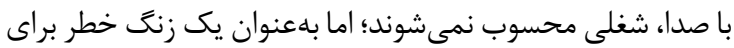

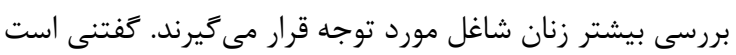

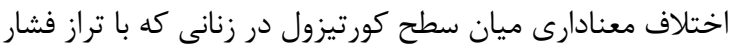

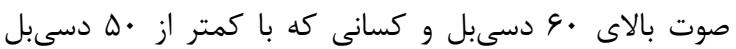

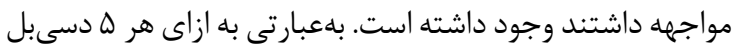

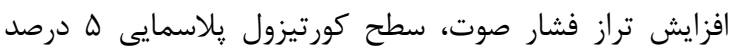

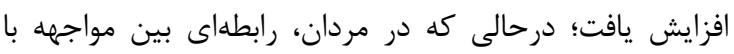

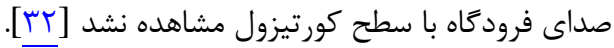

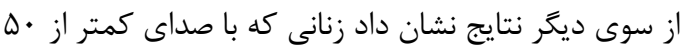

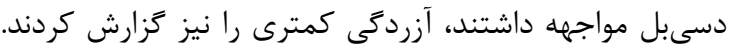

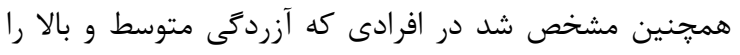

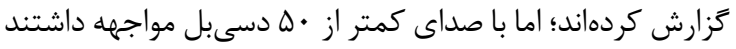

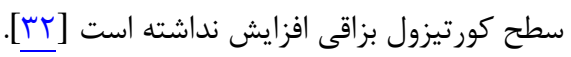
بيان علت اختلاف تأثير صدا بر سطح كورتيزول بزاقى در زنان ندان

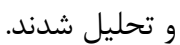

\section{مواد و روشها}

در اين مطالعه، دادههاى مورد نياز از منابع و بانكهاى اطلاعاتى الكترونيكى در دسترس با جستجوى كليد وازمهاى داى دهاري

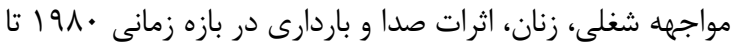

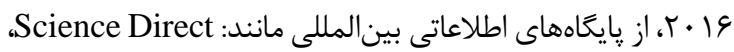
Pubmed ،ISI ،Scopus

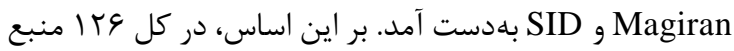

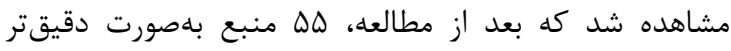

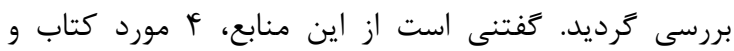

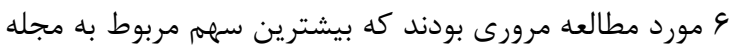
Noise and Health

\section{يافتهها}

براساس يافتههاى بلهدست آمده، اثرات صدا بر سلامتى انسان

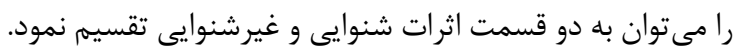

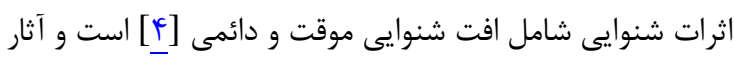

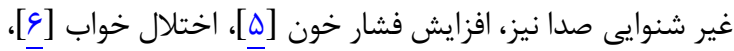

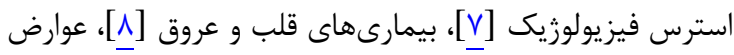

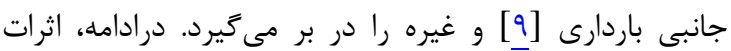
مختلف صدا بر سلامت زنان مرور مى شود.

افت شنواييى

گسترش افت شنوايى به ويزگى هاى صدا همجون: تراز صدا،

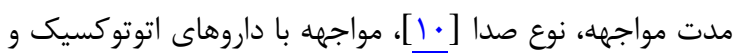

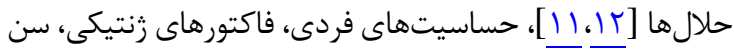

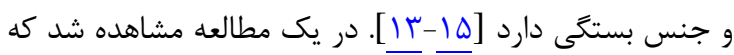

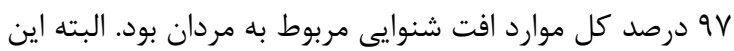

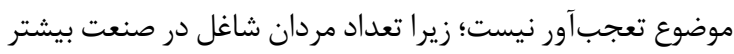

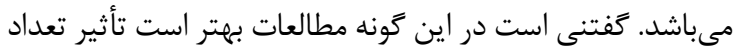
مواجهات، كنترل شود.

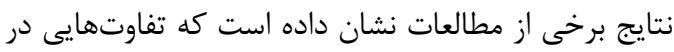

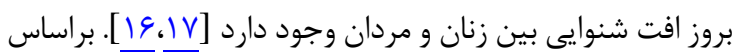

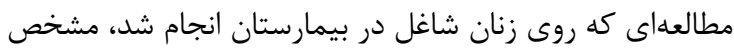

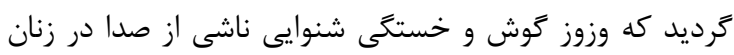

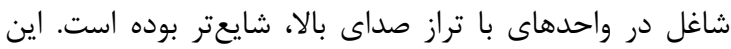

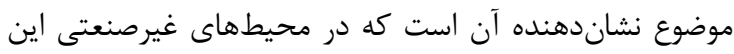
ترازها مى تواند براى زنان مضر باشد [1/1].].

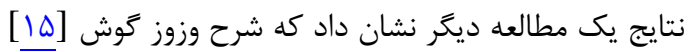

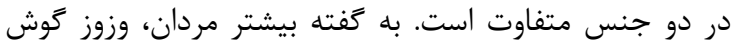

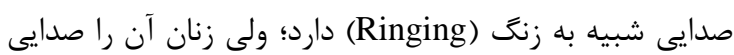

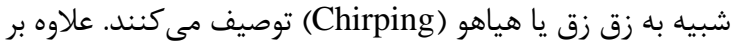

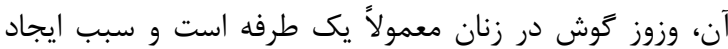


نمى توان كَفت صدا در خه مرحلهاى از دوره باردارى بر جنين تأثير مى كذارد [9].

مطالعات انسانى و حيوانى نشان داده است كه ميزان ميرايى

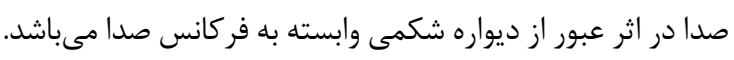

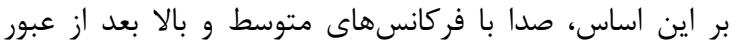

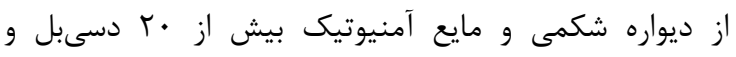

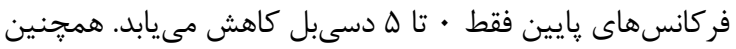

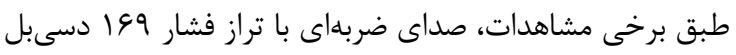

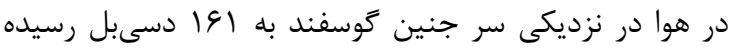

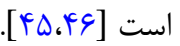

افت شنواييى نوزاد

نتايج مطالعه Cohort در سوئد روى 90 ( • بrا تولد، بين

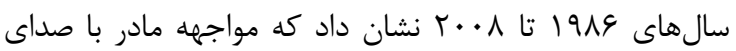
بالاى bم دسىبل در دوران باردارى با اختلال شنوايى كودى

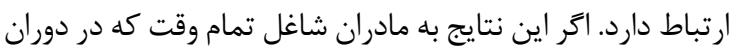

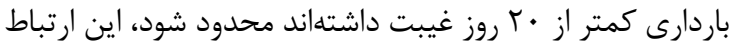

\section{كاهش وزن نوزاد}

نتايج اين مطالعه نشان داد كه مواجهه با صدا باعث افزايش استرس مىشود. براساس يك فرضيه مطرحشده، استرس مى دوانواند

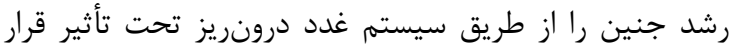

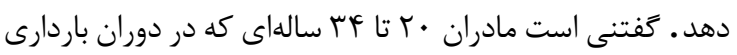
نزديك فرودگاه زندگى مى كردند نوزادانى با وزن كمتر به داس دنيا

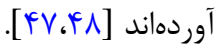

عوارض شنوايى مواجهه باصدا در هر دو گروه زنان و مردان به خوبى شناخته شده است. طبق برخى مطالعات انجامشده،

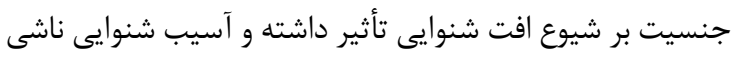

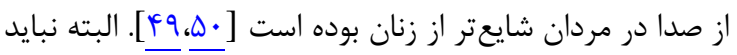

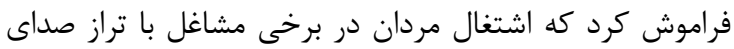

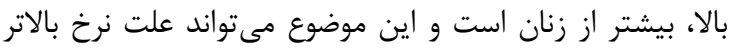

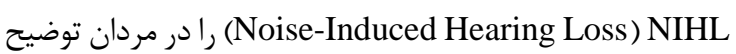

دهد [01]

در برخى مطالعات از صدا بهعنوان عامل استرسزا نام برده شده است كه مىتواند باعث اختلال در مقدار هورمون كورتيزول

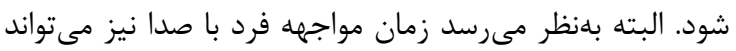

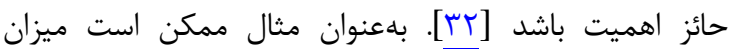

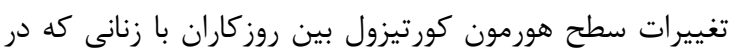
شيفت شب با صدا مواجهه داشتهاند متفاوت باشد.

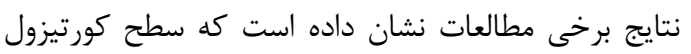
صبحكاهى در زنانى كه با صدا مواجهه دارند، افزايش قابل توجهى نيى
و مردان دشوار است. مطالعات كمى، متغيرهاى تأثيركذار بر

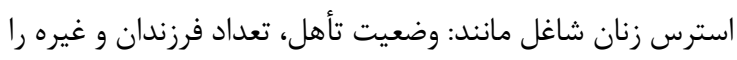

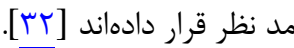

\section{افزايش فشار خون}

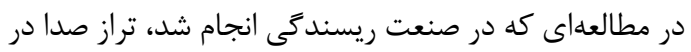

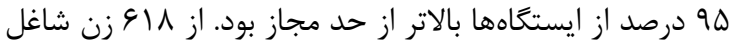

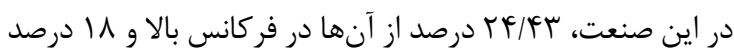

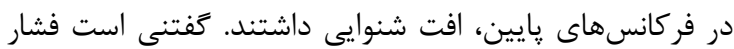

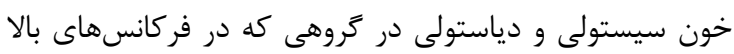

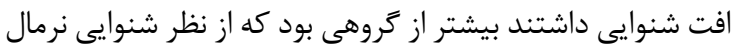

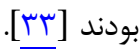
شواهدى وجود دارد كه نشان مىدهد مواجهه مادر با صدا

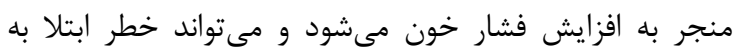
عوارض جانبى باردارى را افزايش دهد [هـ].

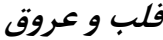

نتايج مطالعات انجامشده نشان مى دهد كه مكانيسمهاى

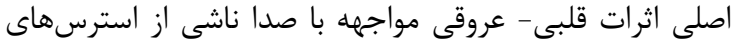

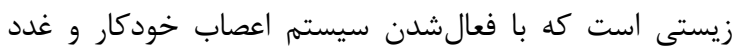

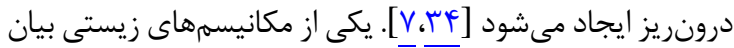

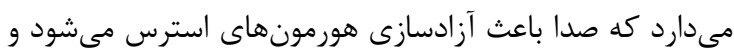

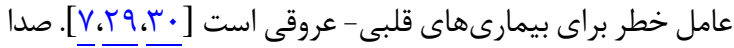

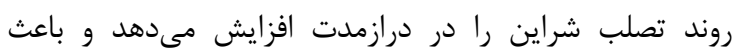

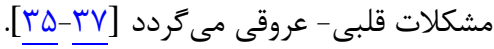
بلنظر مىرسد زنان بيشتر از مردان مردان تحت تأثير عوارض مواجهه با صداى شغلى باشند. در مطالعهاى كه از نظر اثرات صدان

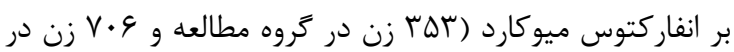

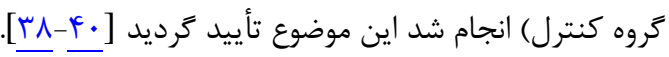

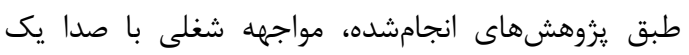

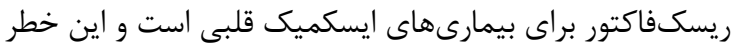

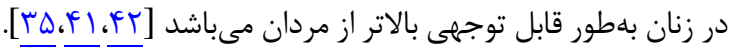

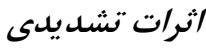
مواجهه همزمان زنان با صدا و شرايط كارى كرم و سرد در

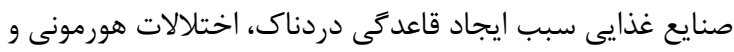

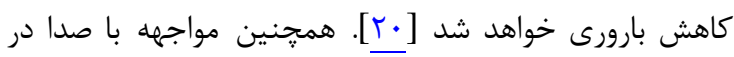

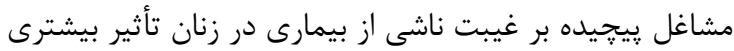

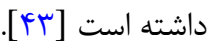

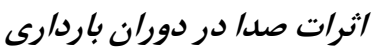

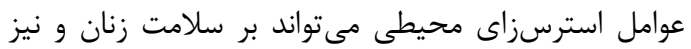

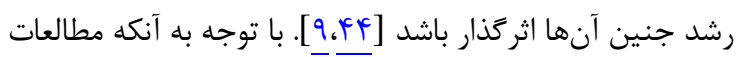
زيادى در مورد اثرات صدا بر جنين انجام نشده است دقيقاً 


$$
\begin{aligned}
& \text { در ايتاليا دستورالعملهاى ارزيابى ريسك مواجهه زنان باردار } \\
& \text { با صدا، ارتعاش و برخى داروها تدوين شده است. }
\end{aligned}
$$

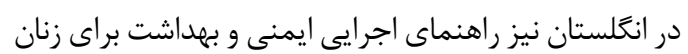

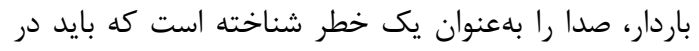

$$
\begin{aligned}
& \text { ارزيابى ريسكها لحاظ شود [بTد]، }
\end{aligned}
$$$$
\text { نتيجه تيرى }
$$

مطالعه مرورى حاضر نشان داد كه مواجهه زنان با صداى

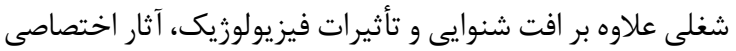

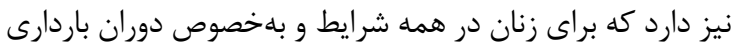

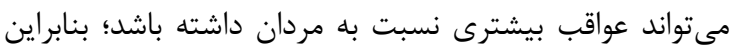

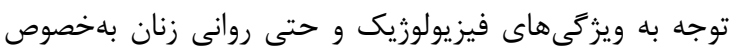

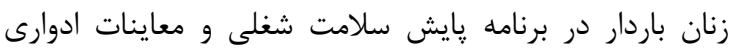

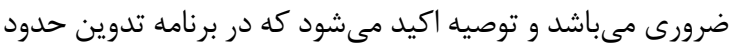
مجاز مواجهه شغلى و يزوهشها به به اين موضوع توجه شود.

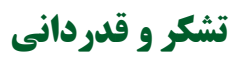

ৎৎ

\section{REFERENCES}

1. Elder S, Schmidt D. Global employment trends for women, 2004. Geneva: Employment Analysis Unit, Employment Strategy Department, International Labour Office; 2004.

2. Frick K. Work environment dialogue in a Swedish municipality-strengths and limits of the Nordic work environment model. Nordic J Work Life Stud. 2013;3(1):69-93.

3. Dzhambov AM, Dimitrova DD. Occupational noise and ischemic heart disease: a systematic review. Noise Health. 2016;18(83):167-77. PMID: 27569404 DOI: 10.4103/14631741.189241

4. Verbeek JH, Kateman E, Morata TC, Dreschler WA, Mischke C. Interventions to prevent occupational noiseinduced hearing loss. Cochrane Database Syst Rev. 2012;10:63-96. PMID: 23076923 DOI: 10.1002/14651858. CD006396.pub3

5. Gehring U, Tamburic L, Sbihi H, Davies HW, Brauer M. Impact of noise and air pollution on pregnancy outcomes. Epidemiology. 2014;25(3):351-8. PMID: 24595395 DOI: 10.1097/EDE.0000000000000073

6. Perron S, Tétreault LF, King N, Plante C, Smargiassi A. Review of the effect of aircraft noise on sleep disturbance in adults. Noise Health. 2012;14(57):58-67. PMID: 22517305 DOI: 10.4103/1463-1741.95133

7. Babisch W. Stress hormones in the research on cardiovascular effects of noise. Noise Health. 2003;5(18):111. PMID: 12631430

8. Babisch W, Houthuijs D, Pershagen G, Katsouyanni K, Velonakis M, Cadum E, et al. Hypertension and exposure to noise near airports-results of the HYENA study. 9th International Congress on Noise as a Public Health Problem (ICBEN), Foxwoods; 2008.

9. Selander J, Albin M, Rosenhall U, Rylander L, Lewne M, Gustavsson P. Maternal occupational exposure to noise during pregnancy and hearing dysfunction in children: a nationwide prospective cohort study in Sweden. Environ Health Perspect. 2016;124(6):855-60. PMID: 26649754 DOI: $10.1289 /$ ehp. 1509874

10. Campo P, Lataye RR. Intermittent noise and equal energy hypothesis. Noise-Induced hearing loss. St. Louis: Mosby Year Book; 1992.

11. Morata TC, Little MB. Suggested guidelines for studying the combined effects of occupational exposure to noise and chemicals on hearing. Noise Health. 2002;4(14):73-87.
داشته؛ اما در مردان ارتباط معنادارى مشاهده نشده است [بr]. اين نتايج مؤيد يك واكنش استرس فيزيولوزيك ناشى از صدا است

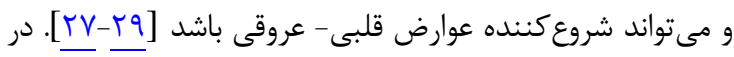
يك مطالعه مرورى سيستماتيك [هَ] كه به بررسى تأثير صدا بر مشكلات قلب و عروق يرداخته است بيان شد كه باوجود اختلافات در روش كار و محدوديتهاى مطالعات مورد بررسى و نتايج ضد و نقيض در آنها، باز هم مى توان از صدا بهعنوان يك ريسكفاكتور

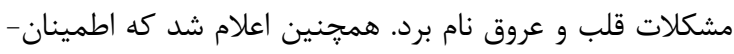
نداشتن در مورد اثر صدا بر سلامت سيستم قلب و عروق در اين گونه مطالعات، نبايد دليل به تعويقانداختن اقدامات ييشخيرانه

$$
\text { براى كارگران بهخصوص زنان باشد. }
$$

امروزه بيشتر زنان در سنين باردارى در بخش صنعت مشغول

به كار هستند و ارتباط بين مواجهه شغلى مادران باردار با صداى بالاى هم دسى بل و اختلال شنوايى در كودكان اثبات شده است [?]]. در برخى از كشورها الزامات خاصى براى محافظت زنان باردار در برابر مواجهات شغلى با صدا وجود دارد. براى مثال:

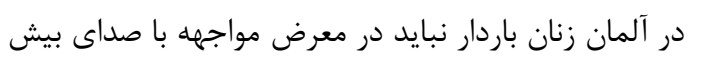

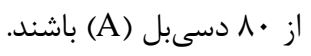

PMID: 12678930

12. Sliwinska-Kowalska M, Zamyslowska-Szmytke E, Szymczak W, Kotylo P, Fiszer M, Wesolowski W, et al. Exacerbation of noise-induced hearing loss by co-exposure to workplace chemicals. Environ Toxicol Pharmacol. 2005;19(3):547-53. PMID: 21783525 DOI: 10.1016/j. etap.2004.12.018

13. Humes LE. Noise-induced hearing loss as influenced by other agents and by some physical characteristics of the individual. J Acoust Soc Am. 1984;76(5):1318-29. PMID: 6512095

14. Pyykkö I, Pekkarinen J, Starck J. Sensory-neural hearing loss in forest workers. An analysis of risk factors. Int Arch Occup Environ Health. 1986;59:439-54.

15. Barrenäs $M$, Luxon L. Pigmentation and noiseinduced hearing loss: is the relationship between pigmentation and noise-induced hearing loss due to an otoxic pheolaminin interaction or to otoprotective eumelan effects. Adv Noise Res. 1998;1:59-71.

16. Fredriksson S, Hammar O, Torén K, Tenenbaum A, Waye KP. The effect of occupational noise exposure on tinnitus and sound-induced auditory fatigue among obstetrics personnel: a cross-sectional study. BMJ Open. 2015;5(3):e005793. PMID: 25818267 DOI: 10.1136/bmjopen-2014-005793

17. Berger EH, Royster LH, Thomas WG. Presumed noiseinduced permanent threshold shift resulting from exposure to an A-weighted Leq of 89 dB. J Acoust Soc Am. 1990; 64(1):192-7. PMID: 711994

18. Estola-Partanen M. Muscular tension and tinnitus: an experimental trial of trigger point injections on tinnitus. [ $\mathrm{PhD}$ Dissertation]. Kirjapaino: Medical School, Department of Otorhinolaryngology, Tampere University Hospital; 2000.

19. Melamed S, Fried Y, Froom P. The joint effect of noise exposure and job complexity on distress and injury risk among men and women: the cardiovascular occupational risk factors determination in Israel study. J Occup Environ Med. 2004;46(10):1023-32. PMID: 15602176

20. Houtman IL, Kauppinen K, Kumpulainen R, Goudswaard A. Gender issues in safety and health at work: a review. Bilbao, Spain: European Agency for Safety and Health at Work; 2003.

21. Carter N, Beh HC. The effect of intermittent noise on cardiovascular functioning during vigilance task performance. Psychophysiology. 1989;26(5):548-59. DOI: 
10.1111/j.1469-8986.1989.tb00708.x

22. Tafalla R, Evans GW. Noise, physiology, and human performance: the potential role of effort. J Occup Health Psychol. 1997;2(2):148-55. PMID: 9552287

23. Evans GW, Lercher P, Meis M, Ising H, Kofler WW. Community noise exposure and stress in children. J Acoust Soc Am. 2001;109(3):1023-7. PMID: 11303916

24. Miki K, Kawamorita K, Araga Y, Musha T, Sudo A. Urinary and salivary stress hormone levels while performing arithmetic calculation in a noisy environment. Ind Health. 1998;36(1):66-9. PMID: 9473861

25. Schulz P, Kirschbaum C, Pruessner J, Hellhammer D. Increased free cortisol secretion after awakening in chronically stressed individuals due to work overload. Stress Health. 1998;14(2):91-7.

26. Hofman LF. Human saliva as a diagnostic specimen. $J$ Nutrition. 2001;131(5):1621S-5. PMID: 11340128

27. Spreng M. Possible health effects of noise induced cortisol increase. Noise Health. 2000;2(7):59-64. PMID: 12689472

28. Babisch W, Fromme H, Beyer A, Ising H. Increased catecholamine levels in urine in subjects exposed to road traffic noise: the role of stress hormones in noise research. Environ Int. 2001;26(7-8):475-81. PMID: 11485215

29. Ising H, Kruppa B. Health effects caused by noise: evidence in the literature from the past 25 years. Noise Health. 2004;6(22):5-13. PMID: 15070524

30. Spreng M. Possible health effects of noise induced cortisol increase. Noise Health. 2000;2(7):59-64. PMID: 12689472

31. Smyth J, Ockenfels MC, Porter L, Kirschbaum C, Hellhammer DH, Stone AS. Stressors and mood measured on a momentory basis are associated with salivary cortisol secretion. Psychoneuroendocrinology. 1998;23(4):353-70. PMID: 9695136

32. Selander J, Bluhm G, Theorell T, Pershagen G, Babisch W, Seiffert I, et al. Saliva cortisol and exposure to aircraft noise in six European Countries. Environ Health Perspect. 2009;117(11):1713-7. PMID: 20049122 DOI: 10.1289/ ehp.0900933

33. Ni CH, Chen ZY, Zhou Y, Zhou JW, Pan JJ, Liu N, et al. Associations of blood pressure and arterial compliance with occupational noise exposure in female workers of textile mill. Chin Med J. 2007;120(15):1309-13. PMID: 17711734

34. McEwen BS, Stellar E. Stress and the individual. Mechanisms leading to disease. Arch Intern Med. 1993; 153(18):2093-101. PMID: 8379800

35. Theorell T, Jood K, Järvholm LS, Vingård E, Perk J, Östergren $\mathrm{PO}$, et al. A systematic review of studies in the contributions of the work environment to ischaemic heart disease development. Eur J Public Health. 2016;26(3):470-7. PMID: 27032996 DOI: 10.1093/eurpub/ckw025

36. Kersten N, Backé E. Occupational noise and myocardial infarction: considerations on the interrelation of noise with job demands. Noise Health. 2015;17(75):116-22. PMID: 25774615 DOI: $10.4103 / 1463-1741.153403$

37. Dimitrova T, Karaslavova E. Vibrations in the working environment and risk of acute myocardial infarction. Med Rev. 2008;44(4):54-7.

38. Hallman T, Burell G, Setterlind S, Oden A, Lisspers J. Psychosocial risk factors for coronary heart disease, their importance compared with other risk factors and gender differences in sensitivity. J Cardiovasc Risk. 2001;8(1):39-
49. PMID: 11234725

39. Wamala SP, Mittleman MA, Horsten M, Schenck-Gustafsson $\mathrm{K}$, Orth-Gomer K. Job stress and the occupational gradient in coronary heart disease risk in women. The Stockholm female coronary risk study. Soc Sci Med. 2000;51(4):481-9. PMID: 10868664

40. Korshøj M, Krustrup P, Jespersen T, Søgaard K, Skotte JH, Holtermann A. A 24-h assessment of physical activity and cardio-respiratory fitness among female hospital cleaners: a pilot study. Ergonomics. 2013;56(6):935-43. PMID: 23586528 DOI: $10.1080 / 00140139.2013 .782427$

41. Higgins JP. Thompson SG. Quantifying heterogeneity in a meta analysis. Stat Med. 2002;21(15):395-8. PMID: 12111919 DOI: $10.1002 / \operatorname{sim} .1186$

42. Kersten N, Backé E. Occupational noise and myocardial infarction: Considerations on the interrelation of noise with job demands. Noise Health. 2015;17(75):116-22. PMID: 25774615 DOI: 10.4103/1463-1741.153403

43. Fried Y, Melamed S, Ben-David HA. The joint effects of noise, job complexity, and gender on employee sickness absence: an exploratory study across 21 organizations- the CORDIS study. J Occup Psychol. 2002;75(2):131-44. DOI: 10.1348/09631790260098181

44. Wu TN, Chen LJ, Lai JS, Ko GN, Shen CY, Chang PY. Prospective study of noise exposure during pregnancy on birth weight. Am J Epidemiol. 1996;143(8):792-6. PMID: $\underline{8610689}$

45. Chordekar S, Kriksunov L, Kishon-Rabin L, Adelman C, Sohmer H. Mutual cancellation between tones presented by air conduction, by bone conduction and by non-osseous (soft tissue) bone conduction. Hear Res. 2012;283(1-2):180-4. PMID: 22037489 DOI: 10.1016/j.heares.2011.10.004

46. Gerhardt KJ, Abrams RM. Fetal exposures to sound and vibroacoustic stimulation. J Perinatol. 2000;20(8 Pt 2):S2130. PMID: 11190697

47. Dancause KN, Laplante DP, Oremus C, Fraser S, Brunet A, King S. Disaster-related prenatal maternal stress influences birth outcomes: project ice storm. Early Hum Dev. 2011;87(12):813-20. PMID: 21784587 DOI: 10.1016/j. earlhumdev.2011.06.007

48. Basner M, Brink M, Bristow A, de Kluizenaar Y, Finegold L, Hong J, et al. ICBEN review of research on the biological effects of noise 2011-2014. Noise Health. 2015;17(75):5782. PMID: 25774609 DOI: $10.4103 / 1463-1741.153373$

49. Tak S, Calvert GM. Hearing difficulty attributable to employment by industry and occupation: an analysis of the National Health Interview Survey-U.S., 1997 to 2003. J Occup Environ Med. 2008;50(1):46 -56. DOI: 10.1097/ JOM.0b013e3181579316

50. Bohnker BK, Betts LS, Page JC, Rovig GW, Sack DM. Navy hearing conservation program: 1995-1999 retrospective analysis of threshold shifts for age, sex, and officer/enlisted status. Mil Med. 2004;169(1):73-6. PMID: 14964507

51. Helfer TM, Canham-Chervak M, Canada S, Mitchener TA. Epidemiology of hearing impairment and noise-induced hearing injury among U.S. military personnel, 2003-2005. Am J Prev Med. 2010;38(1S):S71-S7. PMID: 20117602 DOI: 10.1016/j.amepre.2009.10.025

52. Health and safety executive: a guide for new and expectant mothers on work. Bilbao, Spain: European Agency for Safety and Health at Work; 2005. 